\title{
Радиационное повреждение карбид-кремниевых диодов заряженными частицами высоких энергий
}

\author{
(C) А.М. Стрельчук ${ }^{1}$, В.В. Козловский ${ }^{2}$, А.А. Лебедев ${ }^{1}$ \\ ${ }^{1}$ Физико-технический институт им. А.Ф. Иоффе Российской академии наук, \\ 194021 Санкт-Петербург, Россия \\ ${ }^{2}$ Санкт-Петербургский политехнический университет Петра Великого, \\ 195251 Санкт-Петербург, Россия \\ E-mail: anatoly.strelchuk@mail.ioffe.ru
}

(Получена 4 июля 2018 г. Принята к печати 9 июля 2018 г.)

\begin{abstract}
Представлено исследование радиационной стойкости трех типов коммерческих выпрямительных диодов Шоттки на основе карбида кремния $\left(4 H-\mathrm{SiC}\right.$, уровень легирования базового слоя $\left.(3-7) \cdot 10^{15} \mathrm{~cm}^{-3}\right)$ при электронном облучении (с энергиями электронов 0.9 или $3.5 \mathrm{MэB}$ ), а также при протонном облучении (с энергией протонов $15 \mathrm{MэB).} \mathrm{Контролировались} \mathrm{прямые} \mathrm{и} \mathrm{обратные} \mathrm{вольт-амперные} \mathrm{характеристики}$ диодов. В исходном состоянии диоды характеризовались напряжением пробоя $1-2$ кВ и практически идеальной прямой вольт-амперной характеристикой. Установлено, что наиболее чувствительным к облучению и определяющим радиационную стойкость параметром является последовательное сопротивление диодов, растущее почти на 10 порядков и достигающее $10^{9}$ Ом при больших дозах. Пороговые дозы при облучении электронами лежат в пределах $D_{\mathrm{th}} \approx(0.5-2) \cdot 10^{16} \mathrm{~cm}^{-2}$ и зависят от энергии электронов и уровня легирования базового слоя, а при облучении протонами $D_{\mathrm{th}} \approx 5 \cdot 10^{13} \mathrm{~cm}^{-2}$.
\end{abstract}

DOI: $10.21883 /$ FTP.2018.13.46882.8952

\section{1. Введение}

Начиная с первой большой конференции по карбиду кремния в 1959 г., этот полупроводник рассматривался как перспективный материал для приборов с высокой радиационной стойкостью [1]. С тех пор было сделано много наблюдений, касающихся различных аспектов данной проблемы (см. обзор [2]). В частности, значительное внимание уделялось дефектообразованию при облучении карбида кремния, определялась радиационная стойкость приборов на его основе. Однако результаты работ часто противоречивы, вплоть до того, что утверждение о радиационной стойкости $\mathrm{SiC}$ подвергалось сомнению [3]. Частично такое состояние проблемы можно объяснить, по-видимому, тем, что исследования проводились на очень разнородном по качеству материале, изготовленном разными методами, частично - разнородностью критериев оценки радиационной стойкости [2,4-7]. Технологические проблемы в течение многих лет затрудняли получение высококачественного бездефектного карбида кремния, особенно с невысоким уровнем легирования. В настоящее время эту задачу можно считать в значительной степени решенной. На основе $\mathrm{SiC}$ уже сейчас серийно изготавливается ряд приборов, в частности на рынке предлагаются различные типы выпрямительных диодов, что в свою очередь позволяет провести систематические исследования влияния облучения на $\mathrm{SiC}$. Предварительные результаты исследования (одного типа диодов и одного вида электронного облучения) были приведены нами в [3]. В данной работе представлено исследование радиационной стойкости к двум видам электронного и одному виду протонного облучения нескольких типов наиболее востребованных коммерческих выпрямитель- ных диодов Шоттки на основе $\mathrm{SiC}$ (с напряжениями пробоя $1-2$ кВ).

\section{2. Условия эксперимента}

Исследовались три типа коммерческих диодов Шоттки (SD) на основе слабо легированных (базовых) слоев карбида кремния политипа $4 H$-типа проводимости. Диоды различались уровнем легирования базового слоя и площадью; концентрация нескомпенсированных доноров $N_{d}-N_{a}$ (до облучения) и площадь $S$ составляли: $N_{d}-N_{a} \approx(6-7) \cdot 10^{15} \mathrm{~cm}^{-3}$ и $S \approx 4.8 \cdot 10^{-3} \mathrm{~cm}^{2}$ в диодах Шоттки первого типа (SD1), $N_{d}-N_{a} \approx(3-4) \cdot 10^{15} \mathrm{~cm}^{-3}$ и $S \approx 8 \cdot 10^{-3} \mathrm{~cm}^{2}$ в диодах Шоттки второго типа (SD2), $N_{d}-N_{a} \approx 3 \cdot 10^{15} \mathrm{~cm}^{-3}$ и $S \approx 6.7 \cdot 10^{-2} \mathrm{~cm}^{2}$ в диодах Шоттки третьего типа (SD3). Кроме того, известно, что в диодах Шоттки третьего типа использовалась особая структура (так называемая JBS-структура), позволяющая улучшить вольт-амперную характеристику диода при обратном смещении (см., например, [8]). Диоды располагаются на чипах размером, незначительно превышающим размеры диодов. Общее количество исследовавшихся диодов первого типа было $\sim 40$, второго $\sim 30$ и третьего $\sim 10$. Использовались два вида облучения электронами и один - протонами. Облучение электронами с энергией 0.9 МэВ осуществлялось на ускорителе РТЭ-1В, с энергией 3.5 МэВ на ускорителе ЭЛУ-4, облучение протонами с энергией 15 МэВ проводилось на циклотроне МГЦ-20. Как при электронном, так и при протонном облучении дефекты вводились практически равномерно по всему объему не только слабо легированного слоя, но и подложки, толщины которых ( 10 и $\sim 300$ мкм соответственно) 

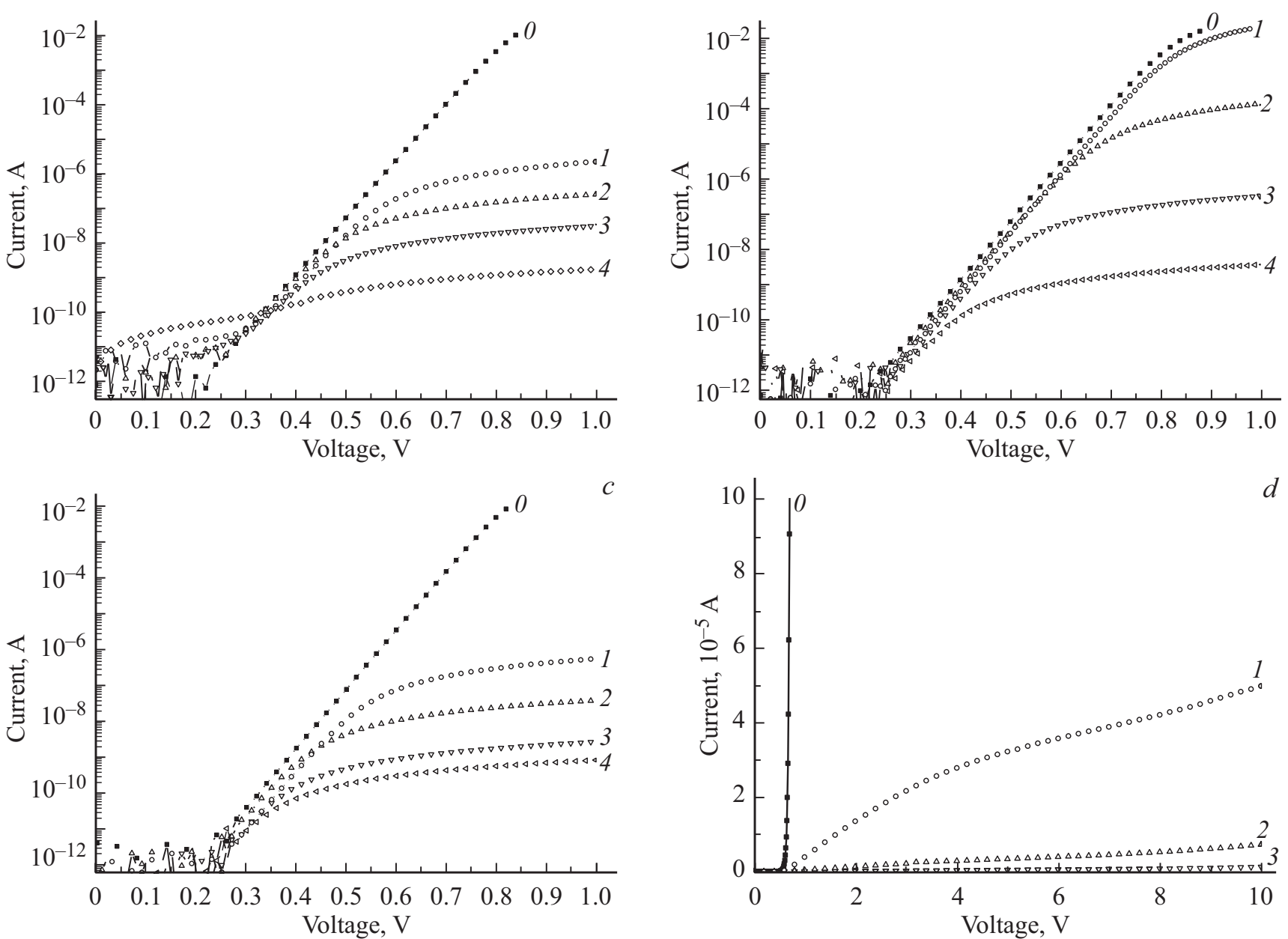

Рис. 1. Прямые вольт-амперные характеристики при комнатной температуре: $a$ - диод Шоттки SD1 до облучения (0, температура $\left.22.2^{\circ} \mathrm{C}\right)$ и после облучения электронами с энергией $0.9 \mathrm{MэB}$ дозой $5 \cdot 10^{16}\left(1,21^{\circ} \mathrm{C}\right), 7 \cdot 10^{16}\left(2,23.5^{\circ} \mathrm{C}\right), 9 \cdot 10^{16}\left(3,23.1^{\circ} \mathrm{C}\right)$, $1.4 \cdot 10^{17} \mathrm{~cm}^{-2}\left(4,23.5^{\circ} \mathrm{C}\right) ; b$ - диод Шоттки SD1 до облучения $\left(0\right.$, температура $\left.21.7^{\circ} \mathrm{C}\right)$ и после облучения протонами с энергией $15 \mathrm{MэB}$, дозой $5 \cdot 10^{13}\left(1,20.8^{\circ} \mathrm{C}\right), 10^{14}\left(2,24^{\circ} \mathrm{C}\right), 1.5 \cdot 10^{14}\left(3,23.7^{\circ} \mathrm{C}\right), 2 \cdot 10^{14} \mathrm{~cm}^{-2}\left(4,22.1^{\circ} \mathrm{C}\right)$; с 一 диод Шоттки $\mathrm{SD} 2$ до облучения $\left(0\right.$, температура $\left.22.4^{\circ} \mathrm{C}\right)$ и после облучения электронами с энергией $0.9 \mathrm{MэВ,} \mathrm{дозой} 5 \cdot 10^{16}\left(1,19.5^{\circ} \mathrm{C}\right), 7 \cdot 10^{16}(2$, $\left.22.5^{\circ} \mathrm{C}\right), 9 \cdot 10^{16}\left(3,21.6^{\circ} \mathrm{C}\right), 1.4 \cdot 10^{17} \mathrm{~cm}^{-2}\left(4,23.7^{\circ} \mathrm{C}\right) ; d-$ то же, что на $a$, но другой диод, без дозы $1.4 \cdot 10^{17} \mathrm{~cm}^{-2}$ и в линейном масштабе.

во всех случаях были значительно меньше длины пробега электронов и протонов (> 1 мм). Во всех случаях облучение проводилось с малым шагом по дозе, что позволяет точнее оценивать пороговые параметры. Вольт-амперные $(I-V)$ характеристики измерялись при комнатной температуре. Последовательное дифференциальное сопротивление в исходных и облученных диодах определялось из вольт-амперных характеристик при прямом смещении и максимальных измеряемых токах (10-20 мА) или напряжениях (10 B).

\section{3. Экспериментальные результаты. Обсуждение}

Для облучения отбирались диоды, характеризующиеся близкой к идеальной $I-V$-характеристикой при прямом смещении, а именно экспоненциальной (до области влияния последовательного сопротивления диода) зависимостью тока от напряжения $I=I_{0} \exp (q V / n k T)$, с коэффициентом идеальности $n \approx 1.02-1.04$ и отсутствием так называемых „утечек“ при малых токах (рис. $1, a-c$, кривые 0). Величина предэкспоненциального множителя составляла $I_{0} \approx(2-3) \cdot 10^{-16} \mathrm{~A}$ при температуре $T=295 \mathrm{~K}$, плотность тока $J_{0} \approx(4-6) \cdot 10^{-14} \mathrm{~A} / \mathrm{cm}^{2}$, для диодов Шоттки первого типа, $I_{0} \approx(4-6) \cdot 10^{-16} \mathrm{~A}$ при $295 \mathrm{~K}$ $J_{0} \approx(5-8) \cdot 10^{-14} \mathrm{~A} / \mathrm{cm}^{2}$ для диодов Шоттки второго типа, $I_{0} \approx 4 \cdot 10^{-15} \mathrm{~A}$ при $294 \mathrm{~K}, J_{0} \approx(5-7) \cdot 10^{-14} \mathrm{~A} / \mathrm{cm}^{2}$ для диодов Шоттки третьего типа. Дифференциальное последовательное сопротивление диодов до облучения находилось в интервале 4-8 Ом. Таким образом, прямые $I-V$-характеристики диодов всех типов до облучения 


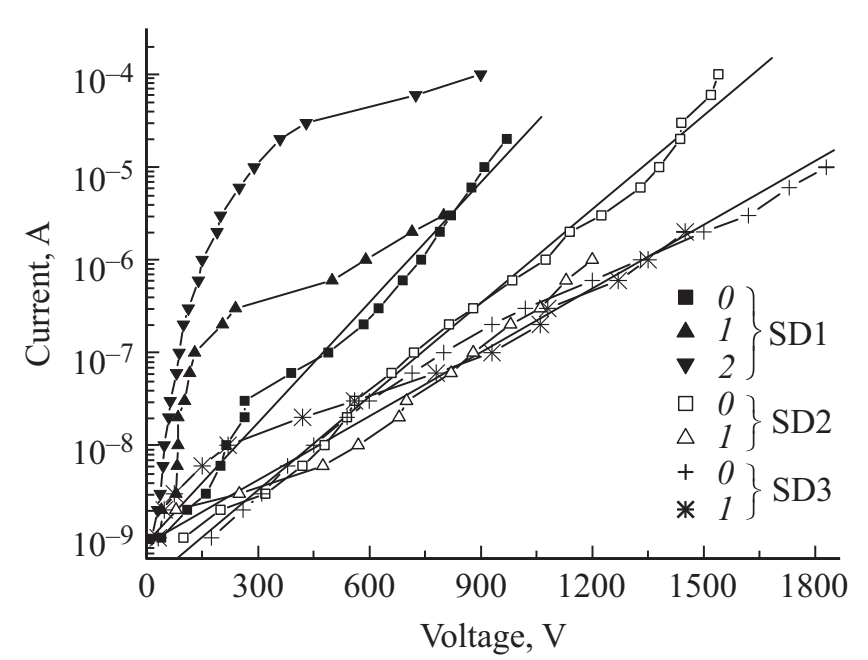

Рис. 2. Обратные вольт-амперные характеристики при комнатной температуре: диоды Шоттки первого (SD1), второго (SD2) и третьего (SD3) типов до облучения (кривые 0) и после облучения электронами с энергией $0.9 \mathrm{MэВ,} \mathrm{дозой}$ $D=5 \cdot 10^{16} \mathrm{~cm}^{-2}$ ( 1 для $\left.\mathrm{SD} 1, \mathrm{SD} 2\right), 3 \cdot 10^{16} \mathrm{~cm}^{-2}$ (1 для SD3), $1.4 \cdot 10^{17} \mathrm{~cm}^{-2}$ (2 для SD1).

оказались близкими друг к другу. Напряжение пробоя до облучения достигало 800-950 В для диодов Шоттки первого типа, 1200-1550 В для диодов Шоттки второго типа и 1600-1860 В для диодов Шоттки третьего типа. Пробой носил „поверхностный“ характер (характеризовался визуально наблюдаемыми искровыми разрядами на поверхности). Предпробойная $I-V$-характеристика для всех типов структур близка к экспоненциальной $I \propto \exp (V / E)$ с характеристическим напряжением $E=100,130$ и 190 В для SD1, SD2 и SD3 соответственно (рис. 2, кривые 0 ).

Облучение диодов Шоттки всех типов в большинстве случаев не изменяет экспоненциальный участок $I-V$-характеристики при прямом смещении (рис. 1,a). Однако в отдельных случаях наблюдался небольшой сдвиг в сторону бо́льших напряжений экспоненциального участка $I-V$-характеристики (на $\sim 15 \mathrm{MB}$, рис. $1, b, c)$. Этот сдвиг не связан с „колебаниями“ комнатной температуры. Кроме того, для диодов Шоттки первого типа как результат облучения зарегистрировано появление омической утечки при малых токах, характеризуемой сопротивлением $4 \cdot 10^{9}$ Ом после облучения электронами с энергией $0.9 \mathrm{MэB,} \mathrm{дозой} 1.4 \cdot 10^{17} \mathrm{~cm}^{-2}$ (рис. $1, a$, кривая 4); в диодах Шоттки второго и третьего типов данный эффект не зарегистрирован.

При малом обратном смещении с увеличением дозы облучения электронами или протонами наблюдалась зависимость обратного тока от дозы разного типа. В частности, в диодах, где до облучения обратный ток не регистрировался, в результате облучения ток появлялся и рос при увеличении дозы. В диодах, где до облучения наблюдался заметный обратный ток, при увеличении дозы ток мог как уменьшаться, так и расти. Напряжение пробоя при облучении электронами с энергией 0.9 МэВ при малых дозах (до $\sim 5 \cdot 10^{16} \mathrm{~cm}^{-2}$ для SD1, SD2 и до $\sim(8-10) \cdot 10^{15} \mathrm{~cm}^{-2}$ для SD3) не изменялось, а при больших дозах начинало уменьшаться (до 750-800 и до $1200 \mathrm{~B}$ для диодов Шоттки первого и второго типов соответственно при дозах $D>5 \cdot 10^{16} \mathrm{~cm}^{-2}$; до 1450 В для диодов Шоттки третьего типа при дозе $\left.D \approx 3 \cdot 10^{16} \mathrm{~cm}^{-2}\right)$. „Поверхностный“ характер пробоя при этом сохранялся. Предпробойный ток в диодах Шоттки первого типа заметно возрастал при больших дозах электронного облучения (рис. 2, кривые 1,2 для SD1); в диодах Шоттки второго и третьего типов предпробойный ток менялся незначительно и экспоненциальный характер обратной $I-V$-характеристики в первом приближении сохранялся (рис. 2, кривые 1 для SD2 и SD3).

Вид прямой $I-V$-характеристики при больших токах (в области влияния последовательного сопротивления диодов) при наборе дозы после облучения всех видов может быть разным, в частности она может быть линейной, а также (после облучения) сублинейной или меняться с суб- на суперлинейную при увеличении прямого напряжения (рис. $1, d$, кривая 1 ). Общей закономерностью является практически независимость от дозы последовательного сопротивления диодов всех типов до набора некоторой дозы (которую условно можно назвать „пороговой“ дозой $\left.D_{\mathrm{th}}\right)$, а затем достаточно резкий рост (на рис. 3 представлены абсолютные значения сопротивления, поскольку различия в площади диодов несущественны). В ряде случаев зарегистрирован значительный разброс сопротивления диодов одного типа, облученных одинаковой дозой (например, достигающий двух порядков разброс $R_{s}$ diff при облучении электронами с энергией $0.9 \mathrm{MэВ,} \mathrm{дозой} D \approx(4-8) \cdot 10^{16} \mathrm{~cm}^{-2}$ диодов первого и второго типов, а также при облучении протонами дозой $D \approx 9 \cdot 10^{13} \mathrm{~cm}^{-2}$ диодов первого типа). Несмотря на разброс сопротивления, рост последовательного сопротивления диодов $\left(R_{S}\right)$ при дозах, превышающих пороговую, в первом приближении можно характеризовать степенной зависимостью вида $R_{s} \propto D^{m}$ с показателем степени $m \approx 10$ для диодов Шоттки первого и второго типов при облучении „низкоэнергетичными“ электронами (с энергией 0.9 МэВ) и $m \approx 15-17$ в других случаях - при облучении „низкоэнергетичными“ электронами диодов Шоттки третьего типа, „высокоэнергетичными“ электронами, с энергией 3.5 МэВ, диодов Шоттки первого типа и протонами диодов Шоттки первого и второго типов (рис. 3). Для определенности за пороговую дозу $D_{\text {th }}$ можно принять дозу, вычисляемую в двойных логарифмических координатах пересечением линии сильной степенной зависимости в области больших доз с линией, отражающей независимость (или слабую зависимость) последовательного сопротивления от дозы при малых дозах. Величина пороговой дозы зависит как от вида облучения, так и от типа диода Шоттки. Так, для диодов Шоттки первого и второго типов при облучении „низкоэнергетичными“ электрона- 


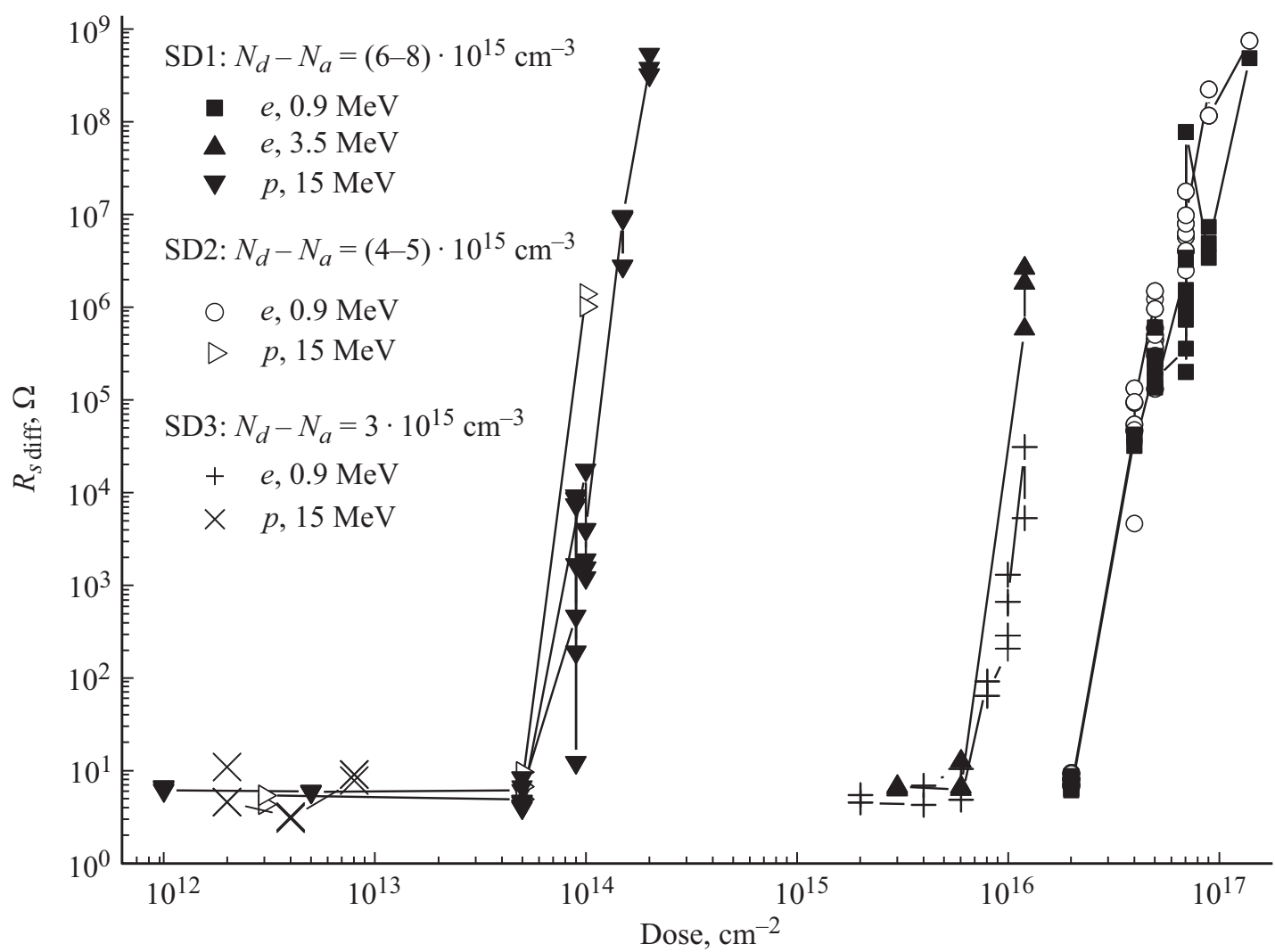

Рис. 3. Зависимости последовательного дифференциального сопротивления диодов Шоттки первого (SD1), второго (SD2) и третьего (SD3) типов от дозы облучения электронами $(e)$ с энергией $0.9,3.5 \mathrm{MэB} \mathrm{и} \mathrm{протонами}(p)$ с энергией 15 МэВ.

ми пороговой является доза $D_{\text {th }} \approx 1.5 \cdot 10^{16} \mathrm{~cm}^{-2}$. Увеличение энергии электронов (до $3.5 \mathrm{MэB}$ ) при облучении диодов первого типа приводит к уменьшению пороговой дозы до $D_{\text {th }} \approx 6 \cdot 10^{15} \mathrm{~cm}^{-2}$. Примерно такой же $\left(D_{\text {th }} \approx 6 \cdot 10^{15} \mathrm{~cm}^{-2}\right)$ является пороговая доза при облучении „низкоэнергетичными“ электронами диодов Шоттки третьего типа, характеризующихся наименьшим в нашем эксперименте уровнем легирования базового слоя. Пороговая доза при облучении протонами (с энергией $15 \mathrm{MэB)} \mathrm{диодов} \mathrm{Шоттки} \mathrm{первого} \mathrm{и} \mathrm{второго} \mathrm{типов}$ характеризуется значением $D_{\text {th }} \approx 5 \cdot 10^{13} \mathrm{~cm}^{-2}$. Данные по пороговым дозам сведены в таблицу. В пределе больших доз, когда $R_{s}$ достигает значения $\sim 1$ ГОм, тенденции к „насыщению“ степенной зависимости $R_{s} \propto D^{m}$ не наблюдается. При увеличении дозы облучения можно предположить дальнейший рост последовательного сопротивления.

Разброс последовательного сопротивления диодов в результате облучения уже наблюдался нами ранее [9]. Было сделано предположение, что разброс $R_{s}$ может быть обусловлен шунтированием основного диода паразитным диодом, связанным с каким-либо дефектом. При таком предположении корректной является оценка последовательного сопротивления диода по его максимальному значению при выбранной дозе. В нашем случае такая поправка не сильно влияет как на характер зависимости сопротивления от дозы (остающейся степенной), так и на величину степени и значение пороговой дозы.

Представленные экспериментальные данные позволяют сделать вывод, что наиболее чувствительным к облучению параметром выпрямительного диода является последовательное сопротивление диода: заметный рост последовательного сопротивления начинается при меньших дозах, чем существенные изменения обратной $I-V$-характеристики. Таким образом, радиационную стойкость исследовавшихся выпрямительных диодов на основе барьера Шоттки можно характеризовать указанными величинами пороговых доз $D_{\text {th }}$. Значение $D_{\text {th }}$ уменьшается при уменьшении легирования базового слоя диода и при увеличении энергии и (или) массы частицы.

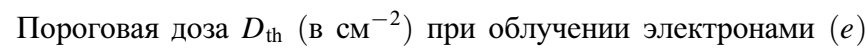
и протонами $(p)$ диодов Шоттки разных типов на основе $4 H-\mathrm{SiC}$

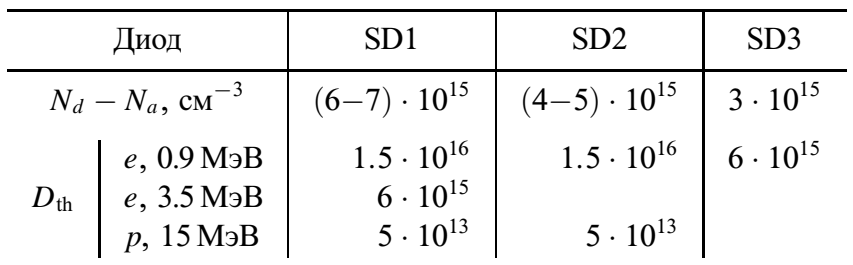


Близкий результат наблюдался при облучении $p$-n-структур и диодов Шоттки на основе карбида кремния протонами, нейтронами и тяжелыми ионами $[10-12]$. В частности, облучение $p-n$-структур на основе $6 \mathrm{H}$-SiC с легированием базового слоя $N_{d}-N_{a} \approx(4-5) \cdot 10^{16} \mathrm{~cm}^{-3}$ протонами с энергией $8 \mathrm{MэB} \mathrm{и} \mathrm{нейтронами} \mathrm{с} \mathrm{энергией} \sim 1$ МэВ приводит к росту последовательного сопротивления диода (до 10 порядков при пороговой дозе $D_{\text {th }} \approx(1-1.5) \cdot 10^{14} \mathrm{~cm}^{-2}$ при облучении протонами $[10]$ и $D_{\text {th }} \approx 2.5 \cdot 10^{15} \mathrm{~cm}^{-2}$ при облучении нейтронами [11]); облучение диодов на основе $4 H$-SiC с легированием базового слоя $N_{d}-N_{a} \approx(5-8) \cdot 10^{15} \mathrm{~cm}^{-3}$ нейтронами с энергией 1 МэВ приводит к росту последовательного сопротивления диода более, чем на 10 порядков, достигая 100 ГОм (пороговая доза $D_{\text {th }} \approx 5 \cdot 10^{13} \mathrm{~cm}^{-2}$ ) [12]. Зависимость последовательного сопротивления от дозы во всех случаях можно представить степенной функцией $R_{s} \propto D^{m}$ с показателем степени $m \approx 7-15$. Предполагалось, что рост последовательного сопротивления диода при увеличении дозы облучения обусловлен ростом сопротивления базового слоя, связанным, по-видимому, в первую очередь с уменьшением концентрации свободных носителей заряда, а также с уменьшением их подвижности.

\section{4. Заключение}

Исследование радиационной стойкости электроннои протонно-облученных коммерческих выпрямительных диодов Шоттки на основе $4 H-\mathrm{SiC}$, рассчитанных на напряжение пробоя 1-2кВ, выявило характер зависимостей тока от напряжения при прямом и обратном смещении и обнаружило ряд эффектов, связанных с облучением. Часть эффектов присуща всем диодам, часть наблюдалась только в отдельных случаях. Установлено, что для всех диодов параметром, наиболее чувствительным к облучению и, следовательно, определяющим радиационную стойкость, является последовательное сопротивление. При этом существует пороговая доза облучения, ниже которой сопротивление не изменяется или изменяется слабо, а при дозах, бо́льших пороговой, последовательное сопротивление диодов резко растет в соответствии со степенной зависимостью с показателем степени от 10 до 17 , достигает значений $\sim 10^{9}$ Ом и не имеет тенденции к насыщению. Пороговая доза при облучении электронами с энергией $0.9-3.5$ МэВ составляет $D_{\text {th }} \approx(0.5-2) \cdot 10^{16} \mathrm{~cm}^{-2}$ и зависит от энергии электронов и уровня легирования базового слоя, а при облучении протонами с энергией $15 \mathrm{MэB} \mathrm{составляет}$ $D_{\mathrm{th}} \approx 5 \cdot 10^{13} \mathrm{~cm}^{-2}$. Напряжение пробоя и допробойный ток менее чувствительны к облучению и начинают заметно изменяться при более высоких дозах.

Работа выполнена при поддержке РНФ (грант № 16-12-10106 „Радиационная стойкость карбида кремния и приборы на его основе для экстремальной электроники“).

\section{Список литературы}

[1] O’Connor, J.R. Smiltens. Proc. Conf. on Silicon Carbide (Pergamon Press, 1960).

[2] Е.В. Калинина. ФТП, 41, 769 (2007).

[3] А.А. Лебедев, К.С. Давыдовская, А.М. Стрельчук, В.В. Козловский. Поверхность. Рентгеновские, синхротронные и нейтронные исследования, 9, 35 (2017).

[4] C. Claes, E. Simoen. Radiation effects in advanced semiconductor materials and devices (Springer Verlag, Berlin Heidelberg, 2002).

[5] E. Omotoso, W.E. Meyer, F.D. Auret, A.T. Paradzah, M. Diale, S.M.M. Coelho, P.J. Janse van Rensburg. Mater. Sci. Semicond. Process., 39, 112 (2015).

[6] А.М. Иванов, Н.Б. Строкан, В.В. Козловский, А.А. Лебедев. ФТП, 42, 370 (2008).

[7] R.K. Sharma, P. Hazdra, S. Popelka. Intern. J. Microelectronics Comput. Sci., 6, 59 (2015).

[8] П.А. Иванов, И.В. Грехов, А.С. Потапов, Н.Д. Ильинская, Т.П. Самсонова, О.И. Коньков. ФТП, 43, 1249 (2009).

[9] A.M. Strel'chuk, V.V. Zelenin, A.N. Kuznetsov, J. Tringe, A.V. Davydov, A.A. Lebedev. Mater. Sci. Forum, 858, 749 (2016).

[10] A.M. Strel'chuk, A.A. Lebedev, V.V. Kozlovski, N.S. Savkina, D.V. Davydov, V.V. Solov'ev, M.G. Rastegaeva. Nucl. Instr. Meth. Phys. Res. B, 147, 74 (1999).

[11] A.M. Strel'chuk, V.T. Gromov, V.V. Zelenin, A.N. Kuznetsov, A.A. Lebedev, N.G. Orlov, N.S. Savkina, V.P. Shukailo. Mater. Sci. Forum, 897, 459 (2017).

[12] Е.В. Калинина, Г.Ф. Холуянов, Д.В. Давыдов, А.М. Стрельчук, А. Hallen, А.О. Константинов, В.В. Лучинин. А.Ю. Никифоров. ФТП, 37, 1260 (2003).

Редактор Л.В. Шаронова

\section{Radiation damage of silicon carbide diodes by high-energy particles}

\author{
A.M. Strel'chuk', V.V. Kozlovski ${ }^{2}$, A.A. Lebedev ${ }^{1}$ \\ ${ }^{1}$ loffe Institute, \\ 194021 St. Petersburg, Russia \\ 2 St. Petersburg State Polytechnic University, \\ 195251 St. Petersburg, Russia
}

\begin{abstract}
The study of radiation hardness of three types of commercial Schottky rectifier diodes based on silicon carbide ( $4 \mathrm{H}-\mathrm{SiC}$, doping level of the base layer is $(3-7) \cdot 10^{15} \mathrm{~cm}^{-3}$ ) under electron irradiation (with electron energies of 0.9 or $3.5 \mathrm{MeV}$ ), as well as proton irradiation (with proton energy of $15 \mathrm{MeV}$ ) is presented. Direct and reverse current-voltage characteristics of the diodes were controlled. In the initial state, the diodes were characterized by a breakdown voltage of $1-2 \mathrm{kV}$ and an almost ideal forward current-voltage characteristic. It was found that the most sensitive to radiation and determining the radiation hardness parameter is the series resistance of the diodes, growing by almost 10 orders of magnitude and reaching $10^{9} \Omega$ at high doses. The threshold doses at electron irradiation lie within $D_{\text {th }} \approx(0.5-2) \cdot 10^{16} \mathrm{~cm}^{-2}$ and depend on the electron energy and doping level of the base layer, and at irradiation by protons $D_{\text {th }} \approx 5 \cdot 10^{13} \mathrm{~cm}^{-2}$.
\end{abstract}

